\title{
Forecasting in the Presence of Structural Breaks and Policy Regime Shifts.
}

\author{
David F. Hendry \\ Economics Department, \\ Oxford University, UK
}

\author{
Grayham E. Mizon* \\ Economics Department, \\ Southampton University, UK
}

September 11, 2001

\begin{abstract}
The value of selecting the best forecasting model as the basis for empirical economic policy analysis is questioned. When no model coincides with the data generation process, non-causal statistical devices may provide the best available forecasts: examples from recent work include intercept corrections and differenced-data VARs. However, the resulting models need have no policy implications. A 'paradox' may result if their forecasts induce policy changes which can be used to improve the statistical forecast. This suggests correcting statistical forecasts by using the econometric model's estimate of the 'scenario' change, and doing so yields reduced biases.
\end{abstract}

\section{Contents}

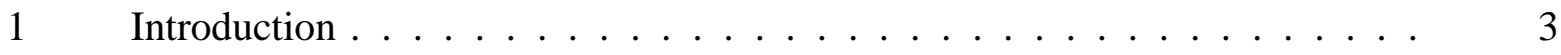

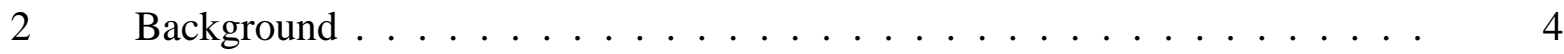

$2.1 \quad$ Forecasting and policy analysis across regime shifts . . . . . . 6

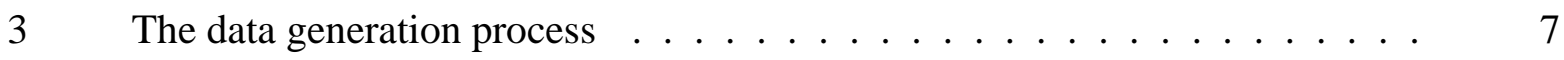

$3.1 \quad$ Forecasting models . . . . . . . . . . . . . . . 8

4 The impacts of breaks on the VEqCM . . . . . . . . . . . . . . . . . . 9

$4.1 \quad$ Breaks in cointegration relations . . . . . . . . . . . . 9

4.2 Post-break forecasts . . . . . . . . . . . . . . . . 11

5 The impacts of breaks on the forecasting model . . . . . . . . . . . . . 12

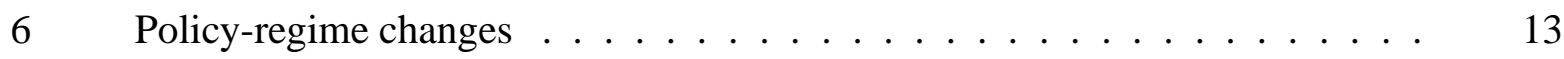

$7 \quad$ Policy-change corrections to robust forecasts . . . . . . . . . . . . 14

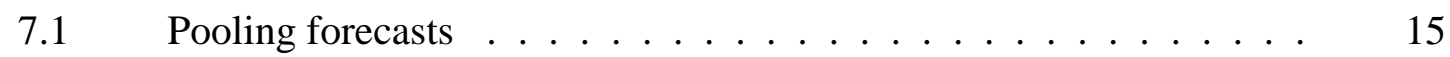

$7.2 \quad$ Intercept corrections . . . . . . . . . . . 15

*Financial support from the UK Economic and Social Research Council under grants R000233447 and L116251015, and the EUI Research Council, is gratefully acknowledged. We are indebted to Anindya Banerjee, Mike Clements, Jurgen Doornik, Neil Ericsson, John Muellbauer, Neil Shephard, Ian Walker and Ken Wallis for helpful comments. 


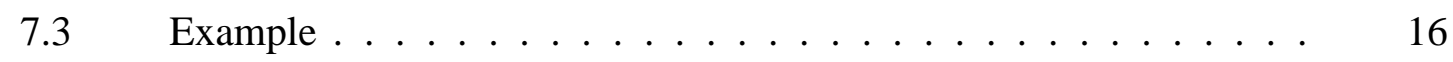

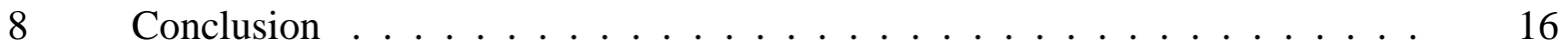

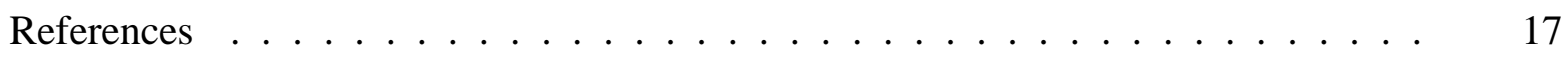




\section{Introduction}

As the title of this volume indicates, the majority of Tom Rothenberg's contributions to econometrics and statistics were in the fields of identification and inference. However, we know that Tom has an interest in all areas of econometrics, even though his awareness of the numerous difficulties to be overcome in undertaking applied work has so far led him to concentrate on theoretical research topics. Therefore, we are delighted to offer our chapter on the use of econometric models in forecasting and economic policy analysis as an indication of some more problems-as well as an analysis of how several of these might be overcome.

A personal anecdote will explain our interest. When the first author reported in Hendry (1981) to the UK Parliamentary Select Committee on the Treasury and Civil Service Enquiry into Monetary Policy, he complained about the proliferation of theoretical models and the dearth of reliable empirical evidence; a decade later in Hendry (1991), he complained to the same committee (now on Official Economic Forecasting) about the abundance of empirical evidence on forecasting - and the absence of theoretical models! We hope to fill a small part of that gap.

In Hendry and Mizon (2000), we investigated three aspects of the relationship between statistical forecasting devices and econometric models in the context of economic policy analysis. First, whether there are grounds for basing economic-policy analysis on the 'best' forecasting system. Second, whether forecast failure in an econometric model precludes its use for economic-policy analysis. Finally, whether in the presence of policy change, improved forecasts can be obtained by using 'scenario' changes derived from the econometric model, to modify an initial statistical forecast. To resolve these issues, we analyzed the problems arising when forecasting takes place immediately after a structural break (i.e., a change in the parameters of the econometric system), but before a regime shift (i.e., a change in the behavior of non-modelled, often policy, variables), perhaps in response to the break (see Hendry and Mizon, 1998, for discussion of this distinction). No forecasting system can be immune to unmodeled breaks that occur after forecasts are announced, whereas some devices are robust to breaks that occur prior to forecasting. These three dichotomies, between econometric and statistical models, structural breaks and regime shifts, and pre and post forecasting events, remain central to our present results. Further particular concerns in the present chapter are working with open models, and considering the effects of structural breaks in cointegrating vectors.

A statistical forecasting device is taken to have no economic-theory basis (in contrast to econometric models for which this is central), so even combined into a system will rarely have implications for economic-policy analysis - and may not even entail links between target variables and policy instruments. This feature will be true of the forecasting models we consider below. Consequently, being the 'best' available forecasting device is insufficient to ensure any value for policy analysis. Some 'forecasting models', such as vector autoregressions (VARs) may also have policy implications, and we comment on such VARs below, but that does not vitiate the previous statement. $^{1}$

The converse is more relevant: does the existence of a dominating forecasting procedure invalidate the use of an econometric model for policy? In Hendry and Mizon (2000), our answer

\footnotetext{
${ }^{1}$ Sims (1986) regards both classes of model distinguished here as 'forecasting' models, but argues that nevertheless both can have policy implications.
} 
was almost the opposite of the Lucas (1976) critique: when forecast failure results from factors unrelated to policy changes - as Stock and Watson (1996) and Clements and Hendry (2001) show often occurs - an econometric model can continue to accurately characterize the response of the economy to the policy, despite its forecast inaccuracy. However, failure may also derive from incorrectly modeled policy reactions.

In this chapter, we show that when forecast failure results from an in-sample structural break induced by a policy-regime shift, forecasts from a statistical model which is robust to the structural break may be improved by combining them with the predicted response from an econometric model of an out-of-sample policy change.

The structure of the chapter is as follows. In the next section, we summarize the relevant forecasting and economic-policy concepts and issues to motivate the analysis, illustrated in section 2.1 by an example of forecasting and policy in the presence of regime shifts. Section 3 formalizes the data generation process, namely a vector equilibrium-correction mechanism, and the forecasting model used as a comparator. Section 4 considers the impact of structural breaks in an open submodel. Then, section 5 investigates the effects of these changes on forecasts from the statistical device, before section 6 describes the policy-scenario changes. Section 7 presents the case for combining the forecasts from robust statistical devices with policy-scenario changes, and contrasts these with pooling and intercept corrections. We present conclusions in section 8 .

\section{Background}

The literature on economic policy is vast, even restricting attention to its implementation to using econometric models: related analyses include Bryant, Hooper and Mann (1993) on evaluating policy regimes; Budd (1998) on conducting economic policy with and without forecasts; Britton (1989) on the more general topic of policy making with macroeconomic models; Sims (1982), Turner, Wallis and Whitley (1989) and Banerjee, Hendry and Mizon (1996) on the econometric analysis of economic policy; and Burns (2001) on the costs of forecast errors in an economicpolicy context. However, the present focus on combining forecasts from models in the face of both deterministic shifts and regime shifts is not prominent in that literature.

The specific rationale for our analysis is as follows. Using the taxonomy of forecast errors in Clements and Hendry (1995), Hendry and Doornik (1997) establish that deterministic shifts are the primary source of systematic forecast failure in econometric models. Deterministic variables include intercepts and linear trends - variables whose future values are known with certainty. Deterministic shifts are viewed as any change in the unconditional expectation of the non-integrated transformations of the variables. In the simplest location model of a variable $y_{t}$ :

$$
y_{t}=\alpha \times 1+u_{t}
$$

with $\alpha \neq 0$ where $\left\{u_{t}\right\}$ is $\mathbf{I}(0)$, a deterministic shift at $T$ is:

$$
y_{T+h}=\alpha^{*} \times 1+u_{T+h} \equiv \alpha \times \mu+u_{T+h}
$$

where $\mu=\alpha^{*} / \alpha \neq 1$ is the shifted intercept. Thus, shifts in parameter of deterministic terms are equivalent to deterministic shifts, as are other factors that mimic deterministic shifts, such as mis-estimating or mis-specifying deterministic components in models. 
The simulation evidence in Hendry (2000) confirms their pernicious effects on forecasts, as well as highlighting the difficulty of detecting other forms of break (i.e., those associated with mean-zero changes). Also, Barrell (2001) illustrates their frequency by considering six major episodes of change during the 1990s. Nevertheless, there exist devices that can robustify forecasting models against such breaks, provided the breaks have occurred prior to forecasting (see e.g., Clements and Hendry, 1996, and Hendry and Clements, 2000): examples include imposing an additional unit root, or adding a specific form of intercept correction. Such 'tricks' can help mitigate forecast failures, but the policy implications of the resulting models are unaltered. That result immediately nullifies the value of judging policy implications by any forecast-based criterion: whether or not a given model forecasts well after a break depends on its robustness to deterministic shifts, not on its 'closeness' to the data generation process.

Importantly, no methods are robust to unanticipated breaks that occur after forecasts are announced, and Clements and Hendry (1999b) show that those same 'robustifying' devices do not offset post-forecasting breaks. However, policy changes that occur post-forecasting will induce breaks in any models that do not embody the appropriate policy links. Thus, such models lose their robustness in that setting. Conversely, despite possibly experiencing forecast failures from pre-forecasting breaks, econometric systems which do embody the relevant policy effects need not experience a post-forecasting break induced by the policy-regime shift. Consequently, when both structural breaks and regime shifts occur, neither class of model alone is adequate: this suggests investigating whether, and if so how, they should be combined. Indeed, the best estimate of the effects of an economic policy change should not be based on the model that is robust to the policy change-its very robustness to the policy change lessens its value for predicting the consequences thereof. Thus, even the existence of a procedure that in the presence of structural breaks and regime shifts systematically produces better forecasts need not invalidate the policy use of another model.

Given the limited knowledge about the data generation process (DGP) that is available to investigators, we adopt a framework in which the DGP is unknown and non-stationary (due to both unit roots and structural breaks), and the econometric model is mis-specified for that DGP. These features seem descriptive of operational economic forecasting, and provide a rationale for using 'intercept corrections' and differencing transformations. A key consequence of these results is that the best available forecasting model need not be based on the 'causal determinants' of the actual economic process, and as the example in $\$ 2.1$ shows, may be based on 'non-causal' variables, that is, variables which do not enter the DGP.

The fact that a purely statistical device may provide the best available forecasts induces an apparent paradox when policy change is feasible. In a world characterized by the framework we adopt, when forecasting after a structural break, forecasts based on the currently-best econometric model may be beaten by statistical devices. Assume for the moment that the statistical forecasting model does not depend on any policy variables, and hence has neither policy implications, nor produces any revisions to its forecasts following policy changes. These 'best' forecasts for some future period are presented to the finance minister of a given country, who thereupon decides that a major policy initiative is essential, and implements it. That the statistical forecasts are not then revised would justifiably be greeted with incredulity. More pertinently, providing the policy model did not fall foul of the critique in Granger and Deutsch (1992), so that changes to policy variables did indeed alter target variables, then a better forecast seems likely by adding the policy change 
effects predicted by the econometric model to the previous forecasts. But this contradicts any claim to the effect that the statistical device produced the best forecasts in a world of structural change and policy-regime shifts.

The resolution depends on distinguishing between unknown, or unanticipated, breaks - where (e.g.) differenced models may deliver the best achievable forecast - and known changes, the consequences of which are partly measurable. The conclusion is that a combination of robustified statistical forecasts with the scenario changes from econometric systems subject to policy interventions may provide improved forecasts. This is the subject of $\S 7$.

\subsection{Forecasting and policy analysis across regime shifts}

Hendry (1997) illustrates the potential role for statistical forecasting methods when an economy is subject to structural breaks, and the econometric model is mis-specified for the DGP. He considers an artificial economy where gross national product (GNP, denoted by $y$ ) is 'caused' solely by the exchange rate $\left(e_{t}\right)$ over a sample prior to forecasting, then the DGP changes to one in which $y$ is only caused by the interest rate $\left(r_{t}\right)$, but this switch is not known by the forecaster. The DGP is non-dynamic, and in particular, the lagged value of $y$ does not affect its behavior (i.e., $y_{t-1}$ is non-causal). Nevertheless, when forecasting after the regime change, on the criterion of forecast unbiasedness, a forecasting procedure that ignores the information on both causal variables and only uses $y_{t-1}$ (namely predicting zero change in $y$ by $\mathrm{E}\left[y_{t} \mid y_{t-1}\right]=y_{t-1}$ ) can have smaller bias than forecasts from models which include the correct causal variable. Here though, neither the statistical model, nor the econometric model based on past causal links, is useful for policy.

Since policy analysis conducted on an incorrect model is rarely useful, we now consider what can be concluded in general settings. The paradigmatic example we have in mind is an econometric model of (say) the tax and benefits system which accurately portrays the relevant links, and yields a good approximation to the changes in revenues and expenditures resulting from changes in the basic rates. Thus, its conditional predictions are accurate. However, it would not necessarily provide good time-series forecasts in an economy subject to structural breaks that affected macroeconomic variables such as total consumers' expenditure and inflation.

The policy implications of any given model in use may or may not change with a particular regime shift. For the setting above, if the exchange rate did not alter when the interest rate was changed in the first regime, so $r_{t}$ had no direct or indirect effect on $y$ in that regime, then the policy implications of the first-regime model would be useless in the second regime. That seems unlikely here, though that might happen in practice. If $e_{t}$ is altered by changes in $r_{t}$, so will $y_{t}$ in both regimes. Policy analysis involves estimation of the target-instrument responses, which in this case means $\partial y_{t+h} / \partial r_{t}$ when $y_{t}$ is the target variable and $r_{t}$ the policy instrument changed at time $t$ when the focus is the effect $h$ periods later. For the statistical model $\Delta y_{t}=\varsigma_{t}$, this response is zero at all forecast horizons $h$, and so despite its robust forecasting abilities, such a model is uninformative for policy analysis. The first-regime econometric model, on the other hand, does provide an estimate of $\partial y_{t+h} / \partial r_{t}$ via (e.g.):

$$
\widehat{\frac{\partial y_{t+h}}{\partial r_{t}}}=\sum_{i=0}^{h} \frac{\partial y_{t+h}}{\partial e_{t+i}} \frac{\partial e_{t+i}}{\partial r_{t}}
$$


In regime- 2 , the actual policy response is $\partial y_{t+h} / \partial r_{t}$, so the regime- 1 econometric model policy responses in (3) will be valuable when they have the same sign, and do not over-estimate the response by more than double, whereas the statistical model is always uninformative in that it gives a zero policy response.

The next section formalizes a more general assumed DGP, before establishing results for forecasting in the face of both structural breaks and regime shifts, when the DGP is a cointegrated system dependent on policy-determined variables. In $\S 7$, we explore the possibility that some combination of statistical forecasts and estimated policy responses could dominate either alone.

\section{The data generation process}

The context for our analysis is an unknown in-sample DGP for the $n=n_{1}+n_{2} \mathrm{I}(1)$ variables $\mathbf{x}_{\mathbf{t}}^{\prime}=\left(\mathbf{y}_{\mathbf{t}}^{\prime}, \mathbf{z}_{\mathbf{t}}^{\prime}\right)$, when $\mathbf{y}_{\mathbf{t}}$ includes target variables and $\mathbf{z}_{\mathbf{t}}$ policy instruments, which is subject to both structural breaks and regime shifts as in Hendry and Mizon (2000). In this section, we concentrate on forecasting $\Delta \mathbf{y}_{\mathbf{T}+\mathbf{h}+\mathbf{1}}$ from time $T+h$ when there was an unknown structural break at $t=T$, and a known policy-regime shift at $t=T+h+1$.

The class of DGP used in our analysis is a vector equilibrium correction model (VEqCM):

$$
\Delta \mathrm{x}_{\mathrm{t}}=\boldsymbol{\tau}+\Gamma \Delta \mathrm{x}_{\mathrm{t}-\mathbf{1}}+\boldsymbol{\alpha} \boldsymbol{\beta}^{\prime} \mathrm{x}_{\mathrm{t}-1}+\epsilon_{\mathrm{t}} \text { when } \epsilon_{\mathrm{t}} \sim \mathrm{IN}_{\mathrm{n}}[\mathbf{0}, \Sigma],
$$

$\mathbf{x}_{\mathbf{t}}$ is $n \times 1$, and $\boldsymbol{\alpha}$ and $\boldsymbol{\beta}$ are $n \times r$ of rank $r<n$. For $t<T$, the $\mathrm{I}(0)$ variables $\Delta \mathbf{x}_{\mathbf{t}}$ and $\boldsymbol{\beta}^{\prime} \mathbf{x}_{\mathbf{t}}$ are stationary, so let:

$$
\mathrm{E}\left[\Delta \mathbf{x}_{\mathbf{t}}\right]=\boldsymbol{\gamma} \text { and } \mathrm{E}\left[\boldsymbol{\beta}^{\prime} \mathbf{x}_{\mathbf{t}}\right]=\boldsymbol{\mu} \quad \forall \mathbf{t} .
$$

Thus taking expectations in (4) yields:

$$
\mathrm{E}\left[\Delta \mathbf{x}_{\mathbf{t}}\right]=\boldsymbol{\tau}+\Gamma \mathrm{E}\left[\Delta \mathrm{x}_{\mathbf{t}}\right]+\boldsymbol{\alpha} \mathrm{E}\left[\boldsymbol{\beta}^{\prime} \mathbf{x}_{\mathbf{t}}\right]
$$

so that:

$$
\boldsymbol{\tau}=\left(\mathbf{I}_{\mathbf{n}}-\Gamma\right) \gamma-\boldsymbol{\alpha} \boldsymbol{\mu}
$$

where $E\left[\Delta \boldsymbol{\beta}^{\prime} \mathbf{x}_{\mathbf{t}}\right]=\boldsymbol{\beta}^{\prime} \boldsymbol{\gamma}=\mathbf{0}$. Substituting from (6) into (4):

$$
\Delta \mathrm{x}_{\mathrm{t}}-\gamma=\Gamma\left(\Delta \mathrm{x}_{\mathrm{t}-1}-\gamma\right)+\boldsymbol{\alpha}\left(\boldsymbol{\beta}^{\prime} \mathbf{x}_{\mathrm{t}-1}-\boldsymbol{\mu}\right)+\boldsymbol{\epsilon}_{\mathrm{t}} .
$$

Factorizing gives the in-sample open VEqCM, conditional on $\mathbf{z}_{\mathbf{t}}$, representing the behavior of the private sector $\mathbf{I}(1)$ variables $\mathbf{y}_{\mathbf{t}}$ :

$$
\left(\Delta \mathbf{y}_{\mathbf{t}}-\gamma_{\mathbf{y}}\right)=\boldsymbol{\Pi}\left(\Delta \mathbf{z}_{\mathbf{t}}-\gamma_{\mathbf{z}}\right)+\boldsymbol{\Lambda}\left(\Delta \mathbf{x}_{\mathbf{t}-\mathbf{1}}-\gamma\right)+\boldsymbol{\lambda}\left(\boldsymbol{\beta}^{\prime} \mathbf{x}_{\mathbf{t}-\mathbf{1}}-\boldsymbol{\mu}\right)+\mathbf{u}_{\mathbf{t}}
$$

and the in-sample marginal model of the policy variables $\mathbf{z}_{\mathbf{t}}$ :

$$
\left(\Delta \mathbf{z}_{\mathbf{t}}-\gamma_{\mathbf{z}}\right)=\Gamma_{\mathbf{z}}\left(\Delta \mathbf{x}_{\mathbf{t}-\mathbf{1}}-\gamma\right)+\boldsymbol{\alpha}_{\mathbf{z}}\left(\boldsymbol{\beta}^{\prime} \mathbf{x}_{\mathbf{t}-\mathbf{1}}-\boldsymbol{\mu}\right)+\boldsymbol{\epsilon}_{\mathbf{z}, \mathbf{t}}
$$

when $\Pi=\Sigma_{\mathrm{yz}} \Sigma_{\mathrm{zz}}^{-1}, \Lambda=\left(\Gamma_{\mathrm{y}}-\Pi \Gamma_{\mathrm{z}}\right), \boldsymbol{\lambda}=\left(\boldsymbol{\alpha}_{\mathrm{y}}-\Pi \boldsymbol{\alpha}_{\mathrm{z}}\right)$ with:

$$
\Sigma=\left(\begin{array}{cc}
\Sigma_{\mathrm{yy}} & \Sigma_{\mathrm{yz}} \\
\Sigma_{\mathrm{zy}} & \Sigma_{\mathrm{zz}}
\end{array}\right), \quad \Gamma=\left(\begin{array}{c}
\Gamma_{\mathrm{y}} \\
\Gamma_{\mathrm{z}}
\end{array}\right)=\left(\begin{array}{cc}
\Gamma_{\mathrm{yy}} & \Gamma_{\mathrm{yz}} \\
\Gamma_{\mathrm{zy}} & \Gamma_{\mathrm{zz}}
\end{array}\right), \quad \alpha=\left(\begin{array}{c}
\alpha_{\mathrm{y}} \\
\alpha_{\mathrm{z}}
\end{array}\right), \quad \gamma=\left(\begin{array}{c}
\gamma_{\mathrm{y}} \\
\gamma_{\mathrm{z}}
\end{array}\right)
$$


and:

$$
\left(\begin{array}{c}
\mathbf{u}_{\mathrm{t}} \\
\epsilon_{\mathrm{z}, \mathrm{t}}
\end{array}\right) \sim \mathbb{I N}_{\mathrm{n}}\left[\left(\begin{array}{c}
\mathbf{0} \\
\mathbf{0}
\end{array}\right),\left(\begin{array}{cc}
\Omega & \mathbf{0} \\
\mathbf{0} & \Sigma_{\mathrm{zz}}
\end{array}\right)\right]
$$

in which $\Omega=\left(\Sigma_{\mathrm{yy}}-\Pi \Sigma_{\mathrm{zz}} \Pi^{\prime}\right)$.

Unfortunately, the key to understanding the impact of policy changes in all such formulations is hidden in the implicit parameter links. In particular, as discussed by Hendry and Mizon (2000), if the parameters in (7) and (8) were unconnected, the system would manifest 'policy ineffectiveness', in that only deviations of $\Delta \mathbf{z}_{\mathbf{t}}$ from $\gamma_{\mathbf{z}}$ would have an impact, with changes in $\gamma_{\mathbf{z}}$ having no effect when implemented by keeping $\Delta \mathbf{z}_{t}-\gamma_{\mathbf{z}}$ fixed. If so, only impulse responses would be of interest. However, shifts in $\gamma_{\mathrm{z}}$ are likely to have an impact on $\Delta \mathrm{y}_{\mathrm{t}}$ in practice, and hence we assume $\gamma_{\mathbf{y}}=\gamma_{0}+\Pi \gamma_{\mathbf{z}}$, namely contemporaneous growth co-breaking (see Clements and Hendry, 1999a and Hendry and Mizon, 1998). Then $\mathbf{z}_{\mathrm{t}}$ is both weakly and super exogenous for all the parameters of interest in (7) when $\boldsymbol{\alpha}_{\mathbf{z}}=\mathbf{0}$, in which case $\boldsymbol{\lambda}=\boldsymbol{\alpha}_{\mathbf{y}}$ : this is assumed in the sequel. Thus:

$$
\Delta \mathbf{y}_{\mathrm{t}}=\gamma_{0}+\Pi \Delta \mathrm{z}_{\mathrm{t}}+\Lambda\left(\begin{array}{c}
\Delta \mathrm{y}_{\mathrm{t}-1}-\gamma_{0} \\
\Delta \mathrm{z}_{\mathrm{t}-1}
\end{array}-{ }_{\mathrm{I}_{\mathrm{n}_{2}}}^{\Pi} \gamma_{\mathrm{z}}\right)+\alpha_{\mathrm{y}}\left(\boldsymbol{\beta}^{\prime} \mathbf{x}_{\mathrm{t}-1}-\boldsymbol{\mu}\right)+\mathbf{u}_{\mathrm{t}}
$$

When $\Delta y_{\mathrm{t}}$ and $\Delta \mathrm{z}_{\mathrm{t}}$ have the same growth rate, $\gamma_{\mathbf{0}}=\mathbf{0}$. Further, if $\Gamma_{\mathrm{zy}}=\mathbf{0}$, then $\Delta \mathbf{y}_{\mathrm{t}}$ would not Granger cause $\mathbf{z}_{\mathbf{t}}$, and $\mathbf{z}_{\mathrm{t}}$ would also be strongly exogenous.

The relevant policy instruments (e.g., interest rates and tax rates) are elements of $\mathbf{z}_{\mathbf{t}}$ that are under the control of the policy agency (e.g., a central bank or Treasury). The fact that in modelling $\mathbf{z}_{\mathbf{t}}$ it is treated as I(1) neither implies that it is intrinsically I(1) nor that it cannot be controlled by the policy agency. Further, although (8) is the representation in the econometric model of the evolution of $\mathbf{z}_{\mathbf{t}}$, the marginal process may differ for particular policy rules in operation from time to time (i.e., due to regime shifts): see e.g., Johansen and Juselius (2000).

\subsection{Forecasting models}

To establish the likely effects of structural breaks and policy-regime shifts on alternative forecasts, a wide range of models could be considered. To illustrate the algebra, we first analyze the properties of an open VEqCM, which is also the in-sample DGP and so correctly embodies policy responses over that period. Then we contrast those findings with the outcome when using a diagonal VAR in second differences (denoted DDV), where every forecasting equation has the form:

$$
\widehat{\Delta y_{i, t}}=\Delta y_{i, t-1}
$$

which therefore does not have any policy implications. Clements and Hendry (1999b) show that these predictors have the same forecast biases for breaks that occur after forecasts are announced, but that the DDV is robust to deterministic breaks that have occurred before forecasting: section 4 draws on their approach, extending it to open models and to forecasts of growth rates (rather than levels). In terms of the example in section 7.3 below, we consider forecasting after a regime shift resulting in a change in the appropriate measurement of the opportunity cost of holding money, and so inducing a structural break in models not incorporating this change, but before a future known policy-regime shift. Since the open VEqCM has some response to policy, but the DDV 
does not, such comparisons yield insights into the effects of using robustified forecasting methods, then exploiting policy-change information via an econometric system.

In the present context, a VAR is simply the unrestricted version of the econometric model so is subject to the same drawbacks, namely a lack of robustness to deterministic shifts, exacerbated by the ill-determination of the estimated intercepts - which compound the (small) growth rates with the (potentially large) equilibrium means-see equation (6) above. A VAR in first differences is mis-specified by omitting any cointegration relations, often of central concern in policy, but thereby gains robustness to equilibrium mean shifts (see e.g., Clements and Hendry, 1999a). Although both these models are sometimes viewed as 'statistical forecasting devices' with possible policy implications, and they lie intermediate between the econometric system in (4) below, and the DDV, introducing them adds little to the understanding of the analysis: Clements and Hendry (1999a) analyze their susceptibility to deterministic shifts.

\section{The impacts of breaks on the VEqCM}

Economic policy analysis, including forecasting after a structural break, is undertaken using the open model in (11), which is assumed to be congruent and encompassing (see Hendry, 1995, Mizon, 1995, and Bontemps and Mizon, 2001, for discussion of these concepts) for $t<T$. A policy-regime shift in the DGP will result in a structural break in (7) unless the parameters of the latter are invariant. Thus, we consider both the policy-regime shift and the structural break affecting the system at time $T$. The policy change alters the instruments $\mathbf{z}_{\mathbf{t}}$ to $\mathbf{z}_{\mathbf{t}}^{*}$, by shifting $\gamma_{\mathbf{z}}$ to $\gamma_{\mathbf{z}}^{*}$ for $t=T, T+1, T+2, \ldots$, and hence $\gamma$ shifts to $\gamma^{*}$. At the same time, there is a structural break affecting the cointegration relations, which change from $\boldsymbol{\beta}$ to $\boldsymbol{\beta}^{*}$, with the new equilibrium mean $\mathrm{E}\left[\boldsymbol{\beta}^{* \prime} \mathbf{x}_{\mathbf{t}}^{*}\right]=\boldsymbol{\mu}^{*}$ accompanying the shift in the growth rate for $t>T$. We leave the other parameters unchanged, since shifts in mean-zero $\mathrm{I}(0)$ combinations of variables do not seem to be of primary importance for forecast failure. In this section, we consider the impacts of parameter changes in the VEqCM DGP over the forecast period on the pre-existing model.

Following the parameter changes noted, (11) becomes:

$$
\begin{aligned}
\Delta \mathbf{y}_{\mathbf{T}+\mathbf{j}}^{*}= & \gamma_{\mathbf{0}}+\Pi \boldsymbol{I}_{\mathbf{T}+\mathbf{j}}^{*}+\Lambda\left(\Delta \mathbf{x}_{\mathbf{T}+\mathbf{j}-\mathbf{1}}^{*}-\gamma^{*}\right) \\
& +\boldsymbol{\lambda}\left(\boldsymbol{\beta}^{* \prime} \mathbf{x}_{\mathbf{T}+\mathbf{j}-\mathbf{1}}^{*}-\boldsymbol{\mu}^{*}\right)+\mathbf{u}_{\mathbf{T}+\mathbf{j}} \text { for } \mathbf{j} \geq \mathbf{1} .
\end{aligned}
$$

The forecaster, though aware of the possibility of parameter changes, does not know their new values. As a result, the open VEqCM (11) estimated using data up to $t=T$, and indeed for $t=T+j$ for $j / T$ small, will suffer forecast failure due to the shift in $\boldsymbol{\mu}$ to $\boldsymbol{\mu}^{*}$ and any additional non-zero components deriving from the unmodeled change in the cointegration vectors.

\subsection{Breaks in cointegration relations}

The introduction of breaks in the cointegration relations raises a number of new considerations, depending on whether the impact induces I(0) or I(1) departures. We consider these in turn. 


\section{I(0) cases}

This case includes the change from $\boldsymbol{\beta}$ to $\boldsymbol{\beta}^{*}$ simply being a linear transformation (rotation), so there exists an $r \times r$ matrix $\mathbf{D}$ of rank $r$ such that $\boldsymbol{\beta}^{*^{\prime}}=\mathbf{D} \boldsymbol{\beta}^{\prime}$ with a corresponding change in the equilibrium mean to $\boldsymbol{\mu}^{*}=\mathbf{D} \boldsymbol{\mu}$ : such a situation is essentially one of no change, merely a re-parameterization. Indeed:

$$
\begin{aligned}
\Delta \mathbf{y}_{\mathbf{T}+\mathbf{j}}^{*}= & \gamma_{\mathbf{0}}+\Pi \Delta \mathbf{z}_{\mathbf{T}+\mathbf{j}}^{*}+\Lambda\left(\Delta \mathbf{x}_{\mathbf{T}+\mathbf{j}-\mathbf{1}}^{*}-\gamma^{*}\right) \\
& +\boldsymbol{\lambda}^{*}\left(\boldsymbol{\beta}^{*} \mathbf{x}_{\mathbf{T}+\mathbf{j}-\mathbf{1}}^{*}-\boldsymbol{\mu}^{*}\right)+\mathbf{u}_{\mathbf{T}+\mathbf{j}} \\
= & \gamma_{0}+\Pi \Delta \mathbf{z}_{\mathbf{T}+\mathbf{j}}^{*}+\Lambda\left(\Delta \mathbf{x}_{\mathbf{T}+\mathbf{j}-\mathbf{1}}^{*}-\gamma^{*}\right) \\
& +\boldsymbol{\lambda}^{*}\left(\mathbf{D} \boldsymbol{\beta}^{\prime} \mathbf{x}_{\mathbf{T}+\mathbf{j}-\mathbf{1}}^{*}-\mathbf{D} \boldsymbol{\mu}\right)+\mathbf{u}_{\mathbf{T}+\mathbf{j}} \\
= & \gamma_{\mathbf{0}}+\Pi \Delta \mathbf{z}_{\mathbf{T}+\mathbf{j}}+\boldsymbol{\Lambda}\left(\Delta \mathbf{x}_{\mathbf{T}+\mathbf{j}-\mathbf{1}}^{*}-\gamma^{*}\right) \\
& +\boldsymbol{\lambda}\left(\boldsymbol{\beta}^{\prime} \mathbf{x}_{\mathbf{T}+\mathbf{j}-\mathbf{1}}-\boldsymbol{\mu}\right)+\mathbf{u}_{\mathbf{T}+\mathbf{j}} \text { for } \mathbf{j} \geq \mathbf{1}
\end{aligned}
$$

where $\lambda=\lambda^{*} \mathrm{D}$.

Another class of change in $\boldsymbol{\beta}$ that leaves $\mathrm{I}(0)$ effects is when more cointegration relations occur. Here $r$ changes to $r^{*}$ (say), and so both $\boldsymbol{\lambda}$ and $\boldsymbol{\beta}$ are changed in dimension, but the outcome is the addition of a mean-zero term - which has minimal effects on forecasts. For the case in which $r^{*}>r$ let $\boldsymbol{\lambda}^{*}=\left(\boldsymbol{\lambda}, \boldsymbol{\lambda}^{\dagger}\right), \boldsymbol{\beta}^{*}=\left(\boldsymbol{\beta}, \boldsymbol{\beta}^{\dagger}\right)$ and $\boldsymbol{\mu}^{* \prime}=\left(\boldsymbol{\mu}, \boldsymbol{\mu}^{\dagger}\right)$ so that we can link the DGP and the forecasting model by:

$$
\begin{aligned}
\Delta \mathbf{y}_{\mathbf{T}+\mathbf{j}}^{*}= & \gamma_{\mathbf{0}}+\boldsymbol{\Pi} \Delta \mathbf{z}_{\mathbf{T}+\mathbf{j}}^{*}+\Lambda\left(\Delta \mathbf{x}_{\mathbf{T}+\mathbf{j}-\mathbf{1}}^{*}-\gamma^{*}\right) \\
& +\boldsymbol{\lambda}^{*}\left(\boldsymbol{\beta}^{* \prime} \mathbf{x}_{\mathbf{T}+\mathbf{j}-\mathbf{1}}^{*}-\boldsymbol{\mu}^{*}\right)+\mathbf{u}_{\mathbf{T}+\mathbf{j}} \\
= & \gamma_{\mathbf{0}}+\boldsymbol{\Pi} \Delta \mathbf{z}_{\mathbf{T}+\mathbf{j}}^{*}+\boldsymbol{\Lambda}\left(\Delta \mathbf{x}_{\mathbf{T}+\mathbf{j}-\mathbf{1}}^{*}-\gamma^{*}\right) \\
& +\boldsymbol{\lambda}\left(\boldsymbol{\beta}^{\prime} \mathbf{x}_{\mathbf{T}+\mathbf{j}-\mathbf{1}}^{*}-\boldsymbol{\mu}^{*}\right)+\mathbf{u}_{\mathbf{T}+\mathbf{j}}^{*} \text { for } \mathbf{j} \geq \mathbf{1}
\end{aligned}
$$

with an error:

$$
\mathbf{u}_{\mathbf{T}+\mathbf{j}}^{*}=\mathbf{u}_{\mathbf{T}+\mathbf{j}}+\boldsymbol{\lambda}^{\dagger}\left(\boldsymbol{\beta}^{\dagger \prime} \mathbf{x}_{\mathbf{T}+\mathbf{j}-\mathbf{1}}^{*}-\boldsymbol{\mu}^{\dagger}\right)
$$

which is $I(0)$ with a zero mean.

However, the outcome is less clear when fewer cointegration relations occur over the forecast period, so some existing elements of $\boldsymbol{\beta}$ are eliminated. This is the case that ${ }^{*}<r$, so partitioning $\boldsymbol{\lambda}, \boldsymbol{\beta}$ and $\boldsymbol{\mu}$ into $\left(\boldsymbol{\lambda}_{\mathbf{1}}, \boldsymbol{\lambda}_{\mathbf{2}}\right)=\left(\boldsymbol{\lambda}_{\mathbf{1}}, \mathbf{0}\right),\left(\boldsymbol{\beta}_{\mathbf{1}}, \boldsymbol{\beta}_{\mathbf{2}}\right)$ and $\left(\boldsymbol{\mu}_{\mathbf{1}}, \boldsymbol{\mu}_{\mathbf{2}}\right)$ respectively:

$$
\begin{aligned}
\Delta \mathbf{y}_{\mathbf{T}+\mathbf{j}}^{*}= & \gamma_{\mathbf{0}}+\Pi \boldsymbol{\Pi}_{\mathbf{T}+\mathbf{j}}^{*}+\boldsymbol{\Lambda}\left(\Delta \mathbf{x}_{\mathbf{T}+\mathbf{j}-\mathbf{1}}^{*}-\gamma^{*}\right) \\
& +\boldsymbol{\lambda}_{\mathbf{1}}\left(\boldsymbol{\beta}_{\mathbf{1}}^{\prime} \mathbf{x}_{\mathbf{T}+\mathbf{j}-\mathbf{1}}^{*}-\boldsymbol{\mu}_{\mathbf{1}}\right)+\mathbf{u}_{\mathbf{T}+\mathbf{j}} \quad \text { for } \mathbf{j} \geq \mathbf{1} \\
= & \gamma_{\mathbf{0}}+\Pi \mathbf{H}_{\mathbf{T}+\mathbf{j}}+\boldsymbol{\Lambda}\left(\Delta \mathbf{x}_{\mathbf{T}+\mathbf{j}-\mathbf{1}}^{*}-\gamma^{*}\right) \\
& +\boldsymbol{\lambda}\left(\boldsymbol{\beta}^{\prime} \mathbf{x}_{\mathbf{T}+\mathbf{j}-\mathbf{1}}^{*}-\boldsymbol{\mu}\right)+\mathbf{u}_{\mathbf{T}+\mathbf{j}}^{*} \quad \text { for } \mathbf{j} \geq \mathbf{1}
\end{aligned}
$$

where:

$$
\mathbf{u}_{\mathbf{T}+\mathbf{j}}^{*}=\mathbf{u}_{\mathbf{T}+\mathbf{j}}-\lambda_{\mathbf{2}}\left(\boldsymbol{\beta}_{\mathbf{2}}^{\prime} \mathbf{x}_{\mathbf{T}+\mathbf{j}-\mathbf{1}}^{*}-\boldsymbol{\mu}_{\mathbf{2}}\right) .
$$

This is only $\mathrm{I}(0)$ provided that $\left(\boldsymbol{\beta}_{\mathbf{2}}^{\prime} \mathbf{X}_{\mathbf{T}+\mathbf{j}-\mathbf{1}}^{*}-\boldsymbol{\mu}_{\mathbf{2}}\right)$ remains $\mathrm{I}(0)$, in which case, $\mathbf{u}_{\mathbf{T}+\mathbf{j}}^{*}$ is still an $\mathrm{I}(0)$ error with a zero mean, but such an outcome seems unlikely. Rather, although $\boldsymbol{\lambda}_{\mathbf{2}}$ has become zero in the DGP, the forecasting model retains linear combinations of $I(1)$ variables that are now $I(1)$, and $\boldsymbol{\mu}_{\mathbf{2}}$ has no meaning. Nevertheless, the simulation results in Hendry (2000) suggest that such changes are difficult to detect empirically. 
A further case of interest is when $r$ is unaltered but there is a set of $k$ additional I(1) variables, $\mathbf{w}_{\mathbf{t}}$ say, which are cointegrated within the DGP, but do not enter the forecasting model in any way. In that case, $\mathbf{x}_{\mathbf{t}}^{* \prime}=\left(\mathbf{x}_{\mathbf{t}}^{\prime}, \mathbf{x}_{\mathbf{t}}^{+\prime}\right)$ is of dimension $(n+k)$ and $\boldsymbol{\beta}^{* \prime}=\left(\boldsymbol{\beta}^{\prime}, \boldsymbol{\beta}^{+\prime}\right)$ is an $r \times(n+k)$ matrix. Thus:

$$
\begin{aligned}
\Delta \mathbf{y}_{\mathbf{T}+\mathbf{j}}^{*}= & \gamma_{\mathbf{0}}+\boldsymbol{\Pi} \Delta \mathbf{z}_{\mathbf{T}+\mathbf{j}}^{*}+\boldsymbol{\Lambda}\left(\Delta \mathbf{x}_{\mathbf{T}+\mathbf{j}-\mathbf{1}}^{*}-\gamma^{*}\right) \\
& +\boldsymbol{\lambda}\left(\boldsymbol{\beta}^{* \prime} \mathbf{x}_{\mathbf{T}+\mathbf{j}-\mathbf{1}}^{*}-\boldsymbol{\mu}^{*}\right)+\mathbf{u}_{\mathbf{T}+\mathbf{j}} \\
= & \gamma_{\mathbf{0}}+\boldsymbol{\Pi} \Delta \mathbf{z}_{\mathbf{T}+\mathbf{j}}^{*}+\boldsymbol{\Lambda}\left(\Delta \mathbf{x}_{\mathbf{T}+\mathbf{j}-\mathbf{1}}^{*}-\gamma^{*}\right) \\
& +\boldsymbol{\lambda}\left(\boldsymbol{\beta}^{\prime} \mathbf{x}_{\mathbf{T}+\mathbf{j}-\mathbf{1}}-\boldsymbol{\mu}\right)+\boldsymbol{\lambda}\left(\boldsymbol{\beta}^{+\prime} \mathbf{w}_{\mathbf{T}+\mathbf{j}-\mathbf{1}}-\boldsymbol{\nu}\right)+\mathbf{u}_{\mathbf{T}+\mathbf{j}} \\
= & \gamma_{\mathbf{0}}+\boldsymbol{\Pi} \mathbf{z}_{\mathbf{T}+\mathbf{j}}^{*}+\boldsymbol{\Lambda}\left(\Delta \mathbf{x}_{\mathbf{T}+\mathbf{j}-\mathbf{1}}^{*}-\gamma^{*}\right) \\
& +\boldsymbol{\lambda}\left(\boldsymbol{\beta}^{\prime} \mathbf{x}_{\mathbf{T}+\mathbf{j}-\mathbf{1}}-\boldsymbol{\mu}\right)+\mathbf{u}_{\mathbf{T}+\mathbf{j}}^{*} \text { for } \mathbf{j} \geq \mathbf{1}
\end{aligned}
$$

where:

$$
\mathbf{u}_{\mathbf{T}+\mathbf{j}}^{*}=\mathbf{u}_{\mathbf{T}+\mathbf{j}}+\boldsymbol{\lambda}\left(\boldsymbol{\beta}^{+\prime} \mathbf{w}_{\mathbf{T}+\mathbf{j}-\mathbf{1}}-\boldsymbol{\nu}\right),
$$

which is $\mathrm{I}(0)$ with a zero mean provided both $\boldsymbol{\beta}^{\prime} \mathbf{x}_{\mathbf{t}}$ and $\boldsymbol{\beta}^{+\prime} \mathbf{w}_{\mathbf{t}}$ are $\mathrm{I}(0)$ individually. None of the above types of $\mathrm{I}(0)$ change in $\boldsymbol{\beta}$ is liable tot induce forecast failure, and so they are not considered further below.

\section{I(1) cases}

An important type of break in the cointegration relations that induces I(1) effects arises in the last case when $\boldsymbol{\beta}^{\prime} \mathbf{x}_{\mathbf{t}}^{*}$ and $\boldsymbol{\beta}^{+\prime} \mathbf{w}_{\mathbf{t}}$ are not $\mathbf{I}(0)$ individually, so that the error in (14) becomes

$$
\mathbf{u}_{\mathbf{T}+\mathbf{j}}^{*}=\mathbf{u}_{\mathbf{T}+\mathbf{j}}+\boldsymbol{\lambda}\left(\boldsymbol{\beta}^{+\prime} \mathbf{w}_{\mathbf{T}+\mathbf{j}-\mathbf{1}}-\boldsymbol{\psi}-\boldsymbol{\nu}\right)
$$

Now the system that excludes $\mathbf{w}_{\mathbf{t}}$ will be seriously mis-specified: both $\left(\boldsymbol{\beta}^{\prime} \mathbf{x}_{\mathbf{T}+\mathbf{j}}-\boldsymbol{\mu}\right)$ and $\mathbf{u}_{\mathbf{T}+\mathbf{j}}^{*}$ will be I(1). As a result $\left(\boldsymbol{\beta}^{\prime} \mathbf{X}_{\mathbf{T}+\mathbf{j}}-\boldsymbol{\mu}\right)$ could become large, so that forecast failure should rapidly manifest itself in this case, forcing a revision to the model specification. This case comes closer to the situation considered in the example in section 7.3, in which the additional I(1) variable is $R_{m}$.

A further case arises when the rank remains the same, but now cointegration becomes defined by new coefficients $\boldsymbol{\beta}^{*}$ when the error in (13) becomes

$$
\mathbf{u}_{\mathbf{T}+\mathbf{j}}^{*}=\mathbf{u}_{\mathbf{T}+\mathbf{j}}+\boldsymbol{\lambda}\left(\boldsymbol{\nabla} \boldsymbol{\beta}^{* \prime} \mathbf{x}_{\mathbf{T}+\mathbf{j}-\mathbf{1}}-\nabla \boldsymbol{\mu}^{*}\right),
$$

with $\nabla \boldsymbol{\beta}^{*}=\left(\boldsymbol{\beta}^{*}-\boldsymbol{\beta}\right)$ and $\nabla \boldsymbol{\mu}^{*}=\left(\boldsymbol{\mu}^{*}-\boldsymbol{\mu}\right)$. Although $\left(\boldsymbol{\beta}^{* \prime} \mathbf{x}_{\mathbf{T}+\mathbf{j}-\mathbf{1}}-\boldsymbol{\mu}^{*}\right)$ is $\mathrm{I}(0)$, $\left(\boldsymbol{\beta}^{\prime} \mathbf{X}_{\mathbf{T}+\mathbf{j}-\mathbf{1}}-\boldsymbol{\mu}\right)$ will be $\mathrm{I}(1)$ and so the unmodified system will suffer similar consequences to the I(1) mistake case noted above. Even if the equilibrium mean did not change, so that $\nabla \boldsymbol{\mu}^{*}=\mathbf{0}$, the same problems would arise since $\mathbf{u}_{\mathbf{T}+\mathbf{j}}^{*}$ will be I(1) via $\nabla \boldsymbol{\beta}^{* 1} \mathbf{x}_{\mathbf{T}+\mathbf{j}-\mathbf{1}}$. For the most favorable case for avoiding forecast failure, when $\boldsymbol{\mu}=\mathbf{0}$, the simulation results in Hendry (2000) show quite high power in detecting such changes, at least in moderately large samples.

\subsection{Post-break forecasts}

We consider forecasting $\Delta \mathbf{y}_{\mathbf{T}+\mathbf{h}+\mathbf{1}}$ using information available at $T+h$ for $h \geq 0$ so that there is an in-sample structural break-unknown to the forecasters. Ignoring estimator variances, and 
assuming accurate data, an economist using the open $\mathrm{VEqCM}$ with $\mathbf{z}_{\mathbf{T}+\mathbf{j}+\mathbf{1}}^{*}$ known, would produce the 1-step ahead forecast:

$$
\widehat{\Delta \mathbf{y}^{*}} T+h+1 \mid T+h=\gamma_{\mathbf{0}}+\boldsymbol{\Pi} \Delta \mathbf{z}_{\mathbf{T}+\mathbf{h}+\mathbf{1}}^{*}+\boldsymbol{\Lambda}\left(\Delta \mathbf{x}_{\mathbf{T}+\mathbf{h}}^{*}-\gamma\right)+\boldsymbol{\lambda}\left(\boldsymbol{\beta}^{* \prime} \mathbf{x}_{\mathbf{T}+\mathbf{h}}^{*}-\boldsymbol{\mu}\right)
$$

of $\Delta \mathbf{y}_{\mathbf{T}+\mathbf{h}+\mathbf{1}}^{*}$, which has a forecast error:

$$
\begin{aligned}
\widehat{\mathbf{u}}_{T+h+1 \mid T+h}= & \Delta \mathbf{y}_{\mathbf{T}+\mathbf{h}+\mathbf{1}}^{*}-\widehat{\Delta \mathbf{y}^{*}} \mathbf{T}+\mathbf{h}+\mathbf{1} \mid \mathbf{T}+\mathbf{h} \\
= & \gamma_{\mathbf{0}}+\Pi \Delta \mathbf{z}_{\mathbf{T}+\mathbf{h}+\mathbf{1}}^{*}+\Lambda\left(\Delta \mathbf{x}_{\mathbf{T}+\mathbf{h}}^{*}-\gamma^{*}\right)+\lambda\left(\beta^{* \prime} \mathbf{x}_{\mathbf{T}+\mathbf{h}}^{*}-\boldsymbol{\mu}^{*}\right)+\mathbf{u}_{\mathbf{T}+\mathbf{h}+\mathbf{1}} \\
& -\gamma_{\mathbf{0}}-\Pi \Delta \mathbf{z}_{\mathbf{T}+\mathbf{h}+\mathbf{1}}^{*}-\Lambda\left(\Delta \mathbf{x}_{\mathbf{T}+\mathbf{h}}^{*}-\gamma\right)-\lambda\left(\boldsymbol{\beta}^{\prime} \mathbf{x}_{\mathbf{T}+\mathbf{h}}^{*}-\boldsymbol{\mu}\right) \\
= & \mathbf{u}_{\mathbf{T}+\mathbf{h}+\mathbf{1}}-\Lambda \nabla \gamma^{*}-\lambda \nabla \boldsymbol{\mu}^{*}+\lambda \nabla \boldsymbol{\beta}^{* \prime} \mathbf{x}_{\mathbf{T}+\mathbf{h}}^{*}
\end{aligned}
$$

when $\nabla \boldsymbol{\mu}^{*}=\left(\boldsymbol{\mu}^{*}-\boldsymbol{\mu}\right), \nabla \boldsymbol{\beta}^{*}=\left(\boldsymbol{\beta}^{*}-\boldsymbol{\beta}\right)$ and $\nabla \boldsymbol{\gamma}^{*}=\left(\boldsymbol{\gamma}^{*}-\gamma\right)$. Hence, the conditional mean of the open VEqCM forecast error is:

$$
\mathrm{E}\left[\widehat{\mathbf{u}}_{\mathrm{T}+\mathrm{h}+1 \mid \mathrm{T}+\mathrm{h}} \mid \mathbf{x}_{\mathrm{T}+\mathbf{h}}^{*}, \mathbf{z}_{\mathbf{T}+\mathbf{h}+\mathbf{1}}^{*}\right]=-\Lambda \nabla \gamma^{*}-\lambda \nabla \mu^{*}+\lambda \nabla \boldsymbol{\beta}^{* \prime} \mathbf{x}_{\mathbf{T}+\mathbf{h}}^{*}
$$

and the unconditional :

$$
\mathrm{E}\left[\widehat{\mathbf{u}}_{\mathrm{T}+\mathrm{h}+1 \mid \mathrm{T}+\mathrm{h}}\right]=-\boldsymbol{\Lambda} \boldsymbol{\nabla} \boldsymbol{\gamma}^{*}+\boldsymbol{\lambda}\left(\mathrm{E}\left[\boldsymbol{\beta}^{* \prime} \mathbf{x}_{\mathbf{T}+\mathbf{h}}^{*}-\boldsymbol{\mu}^{*}\right]-\mathrm{E}\left[\boldsymbol{\beta}^{\prime} \mathbf{x}_{\mathbf{T}+\mathbf{h}}^{*}-\boldsymbol{\mu}\right]\right),
$$

from which it follows that:

$$
\mathrm{V}\left[\widehat{\mathbf{u}}_{\mathrm{T}+\mathrm{h}+1 \mid \mathrm{T}+\mathrm{h}} \mid \mathbf{x}_{\mathbf{T}+\mathbf{h}}^{*}, \mathbf{z}_{\mathbf{T}+\mathbf{h}+\mathbf{1}}^{*}\right]=\mathrm{E}\left[\widehat{\mathbf{u}}_{\mathrm{T}+\mathrm{h}+1 \mid \mathrm{T}+\mathrm{h}} \widehat{\mathbf{u}}_{\mathrm{T}+\mathrm{h}+1 \mid \mathrm{T}+\mathrm{h}}^{\prime}\right]=\Omega
$$

and:

$$
\mathrm{V}\left[\widehat{\mathbf{u}}_{\mathrm{T}+\mathrm{h}+1 \mid \mathrm{T}+\mathrm{h}}\right]=\Omega .
$$

In the absence of a structural break, the economist's forecast would be unbiased, but otherwise is shifted by the changes in the parameters of the deterministic variables and the cointegration relation.

\section{The impacts of breaks on the forecasting model}

We now establish the likely effects of structural breaks and policy-regime shifts on the restricted vector autoregression in second-differenced variables (denoted DDV above). It is shown that the DDV can have a smaller forecast bias than the open VEqCM, because it is robust to forecasting after the equilibrium-mean shift, though it will always have a larger forecast-error variance.

The 1-step ahead statistical-model forecast from $T+h$ for $h \geq 0$ using the DDV is:

$$
\widetilde{\Delta \mathbf{y}^{*}}{ }_{T+h+1 \mid T+h}=\Delta \mathbf{y}_{\mathbf{T}+\mathbf{h}}^{*}
$$

which has a forecast error:

$$
\begin{aligned}
\widetilde{\mathbf{u}}_{T+h+1 \mid T+h}= & \Delta \mathbf{y}_{\mathbf{T}+\mathbf{h}+\mathbf{1}}^{*}-\Delta \mathbf{y}_{\mathbf{T}+\mathbf{h}}^{*} \\
= & \gamma_{\mathbf{0}}+\Pi \Delta \mathbf{z}_{\mathbf{T}+\mathbf{h}+\mathbf{1}}^{*}+\Lambda\left(\Delta \mathbf{x}_{\mathbf{T}+\mathbf{h}}^{*}-\gamma^{*}\right)+\lambda\left(\boldsymbol{\beta}^{* \prime} \mathbf{x}_{\mathbf{T}+\mathbf{h}}^{*}-\boldsymbol{\mu}^{*}\right)+\mathbf{u}_{\mathbf{T}+\mathbf{h}+\mathbf{1}} \\
& -\gamma_{\mathbf{0}}-\Pi \Delta \mathbf{z}_{\mathbf{T}+\mathbf{h}}^{*}-\Lambda\left(\Delta \mathbf{x}_{\mathbf{T}+\mathbf{h}-\mathbf{1}}^{*}-\gamma^{*}\right)-\lambda\left(\boldsymbol{\beta}^{* \prime} \mathbf{x}_{\mathbf{T}+\mathbf{h}}^{*}-\boldsymbol{\mu}^{*}\right)-\mathbf{u}_{\mathbf{T}+\mathbf{j}} \\
= & \Delta \mathbf{u}_{\mathbf{T}+\mathbf{h}+\mathbf{1}}+\Pi \Delta^{2} \mathbf{z}_{\mathbf{T}+\mathbf{h}+\mathbf{1}}^{*}+\Lambda \Delta^{2} \mathbf{x}_{\mathbf{T}+\mathbf{h}}^{*}+\lambda \boldsymbol{\beta}^{* \prime} \Delta \mathbf{x}_{\mathbf{T}+\mathbf{h}}^{*}
\end{aligned}
$$


Thus the conditional mean of the DDV forecast error is given by:

$$
\mathrm{E}\left[\widetilde{\mathbf{u}}_{\mathrm{T}+\mathrm{h}+1 \mid \mathrm{T}+\mathrm{h}} \mid \mathbf{x}_{\mathbf{T}+\mathbf{h}}^{*}, \mathbf{z}_{\mathbf{T}+\mathbf{h}+\mathbf{1}}^{*}\right]=\Pi \boldsymbol{\Delta}^{2} \mathbf{z}_{\mathbf{T}+\mathbf{h}+\mathbf{1}}^{*}+\Lambda \Delta^{2} \mathbf{x}_{\mathbf{T}+\mathbf{h}}^{*}+\lambda \boldsymbol{\beta}^{* \prime} \Delta \mathbf{x}_{\mathbf{T}+\mathbf{h}}^{*}
$$

and the unconditional bias:

$$
\mathrm{E}\left[\widetilde{\mathbf{u}}_{\mathbf{T}+\mathbf{h}+1 \mid \mathrm{T}+\mathrm{h}}\right]=\boldsymbol{\Pi E}\left[\Delta^{2} \mathbf{z}_{\mathbf{T}+\mathbf{h}+\mathbf{1}}^{*}\right]+\Lambda \mathrm{E}\left[\Delta^{2} \mathbf{x}_{\mathbf{T}+\mathbf{h}}^{*}\right]+\lambda \mathrm{E}\left[\boldsymbol{\beta}^{* \prime} \Delta \mathbf{x}_{\mathbf{T}+\mathbf{h}}^{*}\right]=\mathbf{0} .
$$

Thus the bias of the DDV forecast is $\mathbf{0}$, whereas the bias of the open VEqCM is (18), though as the elapsed time, $h$, between the structural break and making the forecast increases, it is more likely that the economist will become aware of the break. Nevertheless, it is possible for the DDV to have a much smaller forecast bias than the open VEqCM, though it will always have a higher variance since (22) and (23) imply that the conditional variance of the DDV forecast error is:

$$
\mathrm{V}\left[\widetilde{\boldsymbol{\epsilon}}_{\mathbf{T}+\mathrm{h}+1 \mid \mathrm{T}+\mathrm{h}} \mid \mathbf{x}_{\mathbf{T}+\mathbf{h}}^{*}, \mathbf{z}_{\mathbf{T}+\mathbf{h}+\mathbf{1}}^{*}\right]=2 \Omega .
$$

Under the assumption that the DGP and the econometric model given in (7) and (8) coincide, it is possible to derive forecast error means and variances for a wider range of the forecasting methods than the open VEqCM and the DDV considered above. This was done in Hendry and Mizon (2000) for a similar class of models and so is not repeated here. However, their results emphasized the different susceptibilities of econometric models and robust statistical forecasting devices to the unknown structural breaks and known regime shifts, thereby indicating possibilities for using each to 'correct' the other.

\section{Policy-regime changes}

We next consider forecasting $\Delta \mathbf{y}_{\mathbf{T}+\mathbf{h}+\mathbf{1}}$ in the presence of a policy experiment where an announced policy shift of $\gamma_{\mathbf{z}}^{*}$ to $\gamma_{\mathbf{z}}^{\mathbf{p}}$ takes place at $T+h+1$ which induces a further shift in the equilibrium mean from $\boldsymbol{\mu}^{*}$ to $\boldsymbol{\mu}^{\mathbf{p}}$ and the growth rates to $\gamma^{\mathbf{p}}$. After the policy-regime shift, the $\mathbf{z}_{\mathbf{t}}$ process takes the form:

$$
\begin{aligned}
& \Delta \mathbf{z}_{\mathbf{T}+\mathbf{h}+\mathbf{1}}^{\mathbf{p}}=\gamma_{\mathbf{z}}^{\mathrm{p}}+\Gamma_{\mathbf{z}}\left(\Delta \mathbf{x}_{\mathbf{T}+\mathbf{h}}^{*}-\gamma^{*}\right)+\epsilon_{\mathbf{z}, \mathbf{T}+\mathbf{h}+\mathbf{1}} \\
& \Delta \mathbf{z}_{\mathbf{T}+\mathbf{h}+\mathbf{j}}^{\mathbf{p}}=\gamma_{\mathbf{z}}^{\mathbf{p}}+\Gamma_{\mathbf{z}}\left(\Delta \mathbf{x}_{\mathbf{T}+\mathbf{h}+\mathbf{j}-\mathbf{1}}^{\mathbf{p}}-\gamma^{\mathbf{p}}\right)+\epsilon_{\mathbf{z}, \mathbf{T}+\mathbf{h}+\mathbf{j}} \text { for } \mathbf{j}>\mathbf{1} .
\end{aligned}
$$

Because $\gamma_{\mathbf{y}}^{\mathbf{p}}=\gamma_{\mathbf{0}}+\Pi \gamma_{\mathbf{z}}^{\mathbf{p}}$, this policy change results in:

$$
\begin{aligned}
\Delta \mathbf{y}_{\mathbf{T}+\mathbf{h}+\mathbf{1}}^{\mathbf{p}}= & \gamma_{\mathbf{0}}+\Pi \Delta \mathbf{z}_{\mathbf{T}+\mathbf{h}+\mathbf{1}}^{\mathbf{p}}+\Lambda\left(\Delta \mathbf{x}_{\mathbf{T}+\mathbf{h}}^{*}-\gamma^{*}\right)+\lambda\left(\beta^{* \prime} \mathbf{x}_{\mathbf{T}+\mathbf{h}}^{*}-\boldsymbol{\mu}^{*}\right)+\mathbf{u}_{\mathbf{T}+\mathbf{h}+\mathbf{1}} \\
\Delta \mathbf{y}_{\mathbf{T}+\mathbf{h}+\mathbf{j}}^{\mathbf{p}}= & \gamma_{\mathbf{0}}+\Pi \Delta \mathbf{z}_{\mathbf{T}+\mathbf{h}+\mathbf{j}}^{\mathbf{p}}+\Lambda\left(\Delta \mathbf{x}_{\mathbf{T}+\mathbf{h}+\mathbf{j}-\mathbf{1}}^{\mathbf{p}}-\gamma^{\mathbf{p}}\right) \\
& +\boldsymbol{\lambda}\left(\boldsymbol{\beta}^{* \prime} \mathbf{x}_{\mathbf{T}+\mathbf{h}+\mathbf{j}-\mathbf{1}}^{\mathbf{p}}-\boldsymbol{\mu}^{\mathbf{p}}\right)+\mathbf{u}_{\mathbf{T}+\mathbf{h}+\mathbf{j}} \quad \text { for } \mathbf{j}>\mathbf{1} .
\end{aligned}
$$

The forecasting performance of the open VEqCM and the DDV is now compared with that of a 'scenario-adjusted' DDV forecast which combines information from each. 


\section{Policy-change corrections to robust forecasts}

Any need to combine two disparate models on the same information set is evidence that both are incomplete: see Clements and Hendry (1998). The encompassing principle argues for finding the congruent representation that can explain the failures of both models, but in the short-run doing so may prove infeasible. When the two models are differently susceptible to the causes of predictive failure, certain combinations could be beneficial: however, the relevant combination must reflect the motivation for pooling (namely, to take account of the effects of known breaks), rather than the usual grounds as discussed in (say) Bates and Granger (1969).

The case of interest is when the robust forecast is made from the DDV, and that prompts a policy response to change the provisional setting $\mathbf{z}_{\mathbf{T}+\mathbf{h}+\mathbf{1}}^{*}$ to the actual outcome $\mathbf{z}_{\mathbf{T}+\mathbf{h}+\mathbf{1}}^{\mathbf{p}}$ (reducing the rate of income tax, say, and thus increasing total final expenditure $x$ in the example in section 7.3). However, from (21) it follows that:

$$
{\widetilde{\Delta \mathbf{y}_{T+h+1 \mid T+h}^{p}}}^{p}={\widetilde{\Delta \mathbf{y}_{T+h+1 \mid T+h}}}_{T}=\Delta \mathbf{y}_{\mathbf{T}+\mathbf{h}}^{*}
$$

and so the DDV forecast is unaltered, implying that its forecast error changes one-for-one with the policy change. Thus:

$$
\begin{aligned}
\widetilde{\mathbf{u}^{\mathbf{p}}} & \quad \Delta \mathbf{y}_{\mathbf{T}+\mathbf{h}+1 \mid \mathbf{1}}^{\mathbf{p}}-\Delta \mathbf{y}_{\mathbf{T}+\mathbf{h}}^{*} \\
= & \gamma_{\mathbf{0}}+\boldsymbol{\Pi} \Delta \mathbf{z}_{\mathbf{T}+\mathbf{h}+\mathbf{1}}^{\mathbf{p}}+\boldsymbol{\Lambda}\left(\Delta \mathbf{x}_{\mathbf{T}+\mathbf{h}}^{*}-\gamma^{*}\right)+\boldsymbol{\lambda}\left(\boldsymbol{\beta}^{* \prime} \mathbf{x}_{\mathbf{T}+\mathbf{h}}^{*}-\boldsymbol{\mu}^{*}\right)+\mathbf{u}_{\mathbf{T}+\mathbf{h}+\mathbf{1}} \\
& -\gamma_{\mathbf{0}}-\boldsymbol{\Pi} \Delta \mathbf{z}_{\mathbf{T}+\mathbf{h}}^{*}-\boldsymbol{\Lambda}\left(\Delta \mathbf{x}_{\mathbf{T}+\mathbf{h}-\mathbf{1}}^{*}-\gamma^{*}\right)-\boldsymbol{\lambda}\left(\boldsymbol{\beta}^{* \prime} \mathbf{x}_{\mathbf{T}+\mathbf{h}}^{*}-\boldsymbol{\mu}^{*}\right)-\mathbf{u}_{\mathbf{T}+\mathbf{h}} \\
= & \Delta \mathbf{u}_{\mathbf{T}+\mathbf{h}+\mathbf{1}}+\boldsymbol{\Pi}\left(\Delta \mathbf{z}_{\mathbf{T}+\mathbf{h}+\mathbf{1}}^{\mathbf{p}}-\Delta \mathbf{z}_{\mathbf{T}+\mathbf{h}}^{*}\right)+\Lambda \Delta^{2} \mathbf{x}_{\mathbf{T}+\mathbf{h}}^{*}+\boldsymbol{\lambda} \boldsymbol{\beta}^{* \prime} \Delta \mathbf{x}_{\mathbf{T}+\mathbf{h}}^{*} .
\end{aligned}
$$

Consequently, the expected DDV unconditional forecast error is:

$$
\mathrm{E}\left[\widetilde{\mathbf{u}}_{\mathrm{T}+\mathrm{h}+1 \mid \mathrm{T}+\mathrm{h}}^{\mathrm{p}}\right]=\Pi\left(\mathrm{E}\left[\Delta \mathbf{z}_{\mathbf{T}+\mathbf{h}+\mathbf{1}}^{\mathrm{p}}\right]-\mathrm{E}\left[\Delta_{\mathbf{\mathbf { T }}+\mathbf{h}}^{*}\right]\right)=\Pi\left(\gamma_{\mathbf{z}}^{\mathbf{p}}-\gamma^{*}\right) .
$$

Forecasts from the open VEqCM, on the other hand, are:

$$
\widehat{\Delta \mathbf{y}}_{T+h+1 \mid T+h}=\gamma_{\mathbf{0}}+\boldsymbol{\Pi} \boldsymbol{\Delta} \mathbf{z}_{\mathbf{T}+\mathbf{h}+\mathbf{1}}^{\mathbf{p}}+\boldsymbol{\Lambda}\left(\boldsymbol{\Delta} \mathbf{x}_{\mathbf{T}+\mathbf{h}}^{*}-\gamma\right)+\boldsymbol{\lambda}\left(\boldsymbol{\beta}^{\prime} \mathbf{x}_{\mathbf{T}+\mathbf{h}}^{*}-\boldsymbol{\mu}\right)
$$

so are revised unconditionally by the difference:

$$
\left[{\widehat{\Delta \mathbf{y}_{T+h+1 \mid T+h}^{p}}}^{p} \widehat{\Delta \mathbf{y}}_{T+h+1 \mid T+h}\right]=\boldsymbol{\Pi}\left(\mathrm{E}\left[\Delta \mathbf{z}_{\mathbf{T}+\mathbf{h}+\mathbf{1}}^{\mathbf{p}}\right]-\mathrm{E}\left[\Delta \mathbf{z}_{\mathbf{T}+\mathbf{h}+\mathbf{1}}^{*}\right]\right)
$$

when $\widehat{\Delta \mathbf{y}}_{T+h+1 \mid T+h}^{p}$ denotes the forecast from (7) based on $\mathbf{z}_{\mathbf{T}+\mathbf{h}+\mathbf{1}}^{\mathbf{p}}$ and $\widehat{\Delta \mathbf{y}}_{T+h+1 \mid T+h}$ that based on $\mathbf{z}_{\mathbf{T}+\mathbf{h}+\mathbf{1}}^{*}$. There is no change in the bias relative to the policy-altered data. Consequently, if the policy regime shift at $t=T+h+1$ does not lead to a structural break in $\Pi$, the econometric model would correctly predict the impact of the regime shift, despite the deterministic structural break at $t=T$. This opens up the possibility of a combined forecast performing well. In particular, adding the scenario effect from (29) to the structural break-robust DDV forecast to give a combined forecast of the form:

$$
\overline{\Delta \mathbf{y}}_{T+h+1 \mid T+h}={\widetilde{\Delta \mathbf{y}_{T+h+1 \mid T+h}^{p}}}^{p}\left[{\widehat{\Delta \mathbf{y}_{T+h+1 \mid T+h}^{p}}}^{p}{\widehat{\Delta \mathbf{y}_{T+h+1 \mid T+h}}}\right]
$$


which might avoid much of the structural break, yet capture some, and possibly all, of the policy effect.

The unconditional bias of $\overline{\Delta \mathbf{y}}_{T+h+1 \mid T+h}$ from (30) is:

$$
\begin{aligned}
& \mathrm{E}\left[\Delta \mathbf{y}_{\mathbf{T}+\mathbf{h}+\mathbf{1}}^{\mathrm{p}}-\overline{\Delta \mathbf{y}}_{\mathbf{T}+\mathbf{h}+\mathbf{1} \mid \mathbf{T}+\mathbf{h}}\right]=\mathrm{E}\left[\Delta \mathbf{y}_{\mathbf{T}+\mathbf{h}+\mathbf{1}}^{\mathrm{p}}-{\widetilde{\Delta \mathbf{y}_{\mathbf{T}+\mathbf{h}+\mathbf{1} \mid \mathbf{T}+\mathbf{h}}^{\mathrm{p}}}}_{\mathrm{p}}\right] \\
& -\mathrm{E}\left[{\widehat{\Delta \mathbf{y}_{\mathrm{T}+\mathrm{h}+1 \mid \mathrm{T}+\mathrm{h}}^{\mathrm{p}}}}^{\mathrm{P}} \widehat{\Delta \mathbf{y}}_{\mathrm{T}+\mathrm{h}+1 \mid \mathrm{T}+\mathrm{h}}\right] \\
& =\Pi\left(\gamma_{\mathbf{z}}^{\mathbf{p}}-\gamma^{*}\right)-\Pi\left(\gamma_{\mathbf{z}}^{\mathbf{p}}-\gamma^{*}\right)=\mathbf{0}
\end{aligned}
$$

Thus, in the absence of sampling variability and any model mis-specifications, the scenariocorrected forecast would be unbiased, whereas each model alone would fail on the pre or post forecasting changes.

\subsection{Pooling forecasts}

The outcome in section 7 can differ considerably from what would happen with 'forecast pooling'. We illustrate using the mean of the two forecasts:

$$
\overline{\overline{\Delta \mathbf{y}}}_{T+h+1 \mid T+h}=\frac{1}{2}\left({\widehat{\Delta \mathbf{y}^{\mathbf{p}}}}_{T+h+1 \mid T+h}+{\widetilde{\Delta \mathbf{y}_{T+h+1 \mid T+h}}}\right)
$$

while recognizing that other choices of weighting factors are widely used. The unconditional bias of $\overline{\overline{\Delta \mathbf{y}}}_{T+h+1 \mid T+h}$ from (31) is:

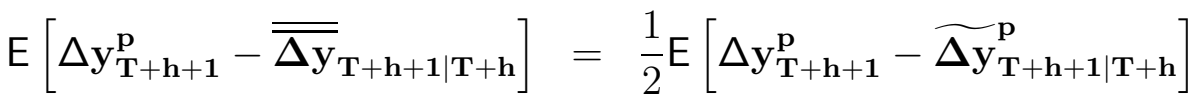

$$
\begin{aligned}
& +\frac{1}{2} \mathrm{E}\left[\Delta \mathbf{y}_{\mathbf{T}+\mathbf{h}+\mathbf{1}}^{\mathbf{p}}-\widehat{\Delta \mathbf{y}}_{\mathbf{T}+\mathbf{h}+\mathbf{1} \mid \mathbf{T}+\mathbf{h}}^{\mathbf{p}}\right] \\
& =\frac{1}{2}\left\{\boldsymbol{\Pi}\left(\gamma_{\mathbf{z}}^{\mathbf{p}}-\boldsymbol{\gamma}^{*}\right)+\left(-\boldsymbol{\Lambda} \nabla \gamma^{*}+\lambda \mathrm{E}\left[\boldsymbol{\nabla} \boldsymbol{\beta}^{* \prime} \mathbf{x}_{\mathbf{T}+\mathbf{h}}^{*}\right]-\nabla \boldsymbol{\mu}^{*}\right)\right\} \\
& \neq \mathbf{0} \text {, }
\end{aligned}
$$

as the bias in the VEqCM forecast is still given by (18):

$$
\begin{aligned}
\mathrm{E}\left[\Delta \mathbf{y}_{\mathbf{T}+\mathbf{h}+\mathbf{1}}^{\mathbf{p}}-\widehat{\Delta \mathbf{y}}_{\mathbf{T}+\mathbf{h}+\mathbf{1} \mid \mathbf{T}+\mathbf{h}}^{\mathbf{p}}\right]= & \gamma_{\mathbf{0}}+\boldsymbol{\Pi E}\left[\Delta \mathbf{z}_{\mathbf{T}+\mathbf{h}+\mathbf{1}}^{\mathbf{p}}\right]+\boldsymbol{\Lambda} \mathrm{E}\left[\Delta \mathrm{x}_{\mathbf{T}+\mathbf{h}}^{*}-\gamma^{*}\right]+\lambda \mathrm{E}\left[\boldsymbol{\beta}^{* \prime} \mathbf{x}_{\mathbf{T}+\mathbf{h}}^{*}-\boldsymbol{\mu}^{*}\right] \\
& -\left(\gamma_{\mathbf{0}}+\boldsymbol{\Pi E}\left[\Delta \mathbf{z}_{\mathbf{T}+\mathbf{h}+\mathbf{1}}^{\mathbf{p}}\right]+\boldsymbol{\Lambda} \mathrm{E}\left[\Delta \mathbf{x}_{\mathbf{T}+\mathbf{h}}^{*}-\gamma\right]+\lambda \mathrm{E}\left[\boldsymbol{\beta}^{\prime} \mathbf{x}_{\mathbf{T}+\mathbf{h}}^{*}-\boldsymbol{\mu}\right]\right) \\
= & -\boldsymbol{\Lambda} \boldsymbol{\nabla} \gamma^{*}+\lambda \mathrm{E}\left[\boldsymbol{\nabla} \boldsymbol{\beta}^{* \prime} \mathbf{x}_{\mathbf{T}+\mathbf{h}}^{*}\right]-\boldsymbol{\nabla} \boldsymbol{\mu}^{*} .
\end{aligned}
$$

Thus, there is little to be gained from average pooling (or related weights), since the requirement of offsetting both the structural break and the regime shift involves all three models, with the pre-regime change forecast entering negatively.

\subsection{Intercept corrections}

The intercept correction we have in mind is simply 'setting the model back on track' immediately prior to the forecast calculation, corresponding to an impulse indicator equal to unity from time $T+h$. This adds the last estimation-sample error to the forecast, which from (18) at time $T+h$ is:

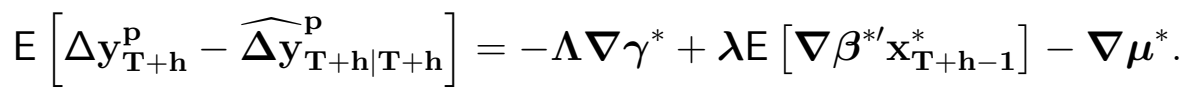


Consequently, most of the break will be corrected, leaving only estimation errors and $\lambda \mathrm{E}\left[\boldsymbol{\nabla} \boldsymbol{\beta}^{* \prime} \boldsymbol{\Delta} \mathrm{x}_{\mathbf{T}+\mathbf{h}}^{*}\right]$ on average. Again, there are variance consequences (the forecast-error variance is doubled for innovation errors), but these remain small relative to the sizes of the breaks discussed in the example in the next section.

\subsection{Example}

A potential example is one in which $\mathbf{y}_{\mathbf{t}}$ includes real money holdings $(m-p)$ (M1), inflation $\Delta p$, and real total final expenditure $x$, whereas $\mathbf{z}_{\mathbf{t}}$ includes the opportunity cost of holding money. The effect of the UK Banking Act of 1984, which made the payment of interest on checking accounts legal, is represented in this framework by a change in the opportunity cost of holding money from $R_{a}$, the return from an alternative asset to money (e.g., the three-month local authority interest rate) to $R_{n}=R_{a}-R_{m}$, when $R_{m}$ is the interest rate paid on checking accounts. Money demand equations based on $R_{a}$ experienced major forecast failure post 1985 as $(m-p)$ increased dramatically, although identical equations using $R_{n}$ remained constant. Models of this aspect of the UK economy have been extensively analyzed by, inter alia, Hendry and Ericsson (1991), Boswijk (1992), Johansen (1992), Hendry and Mizon (1993), Harris (1995), Paroulo (1996), Rahbek, Kongsted and Jørgensen (1999), and Doornik, Hendry and Nielsen (1998).

Forecasting models of the DDV form, such as:

$$
\Delta \widehat{(m-p)_{T+1}}=\Delta(m-p)_{T},
$$

did not suffer forecast failure from 1985 onwards. A government that was concerned about the inflationary consequences of the large increase in M1 might have raised income taxes, providing the combined event of interest.

Some representative orders of magnitude may help to indicate when, and why, the DDV might dominate the VEqCM in forecasting when deterministic and related shifts occur. In many estimated econometric equations, residual standard deviations lie between $0.5 \%$ and $1.5 \%$ of the levels of the dependent variables (albeit that some fall outside this region). Any deterministic shift in excess of $2 \%$ will, therefore, favor the DDV in short-term forecasts. The example of the 1984 Banking Act introducing interest-bearing sight deposits led to a more than $40 \%$ increase in holdings of M1 over a couple of years; the 1986 Building Societies Act (the UK analogue of Savings and Loans Associations in the US) which permitted borrowing on wholesale as well as retail money markets, induced a doubling in mortgage lending over four years. Such massive shifts swamp any uncertainty effects.

\section{Conclusion}

The best forecasting model is not necessarily a good basis for economic-policy analysis. When models are mis-specified for a non-stationary data generation process, non-causal statistical devices may provide the best available forecasts, but need have no policy implications. Moreover intercept corrections act like differencing and improve forecasts without altering policy conclusions. Conversely, forecast failure in an econometric model need not preclude its use for policy analysis, since deterministic shifts need not alter policy reactions.. 
The impacts of a range of structural breaks and policy regime shifts on both forecasting devices are analyzed, and reveals that neither model is immune to both changes. Thus, we investigate correcting the statistical forecast using the econometric model's estimate of the 'scenario' change resulting from the regime shift. The outcome shows a great improvement in robustness, and dominates pooling the two forecasts, and perhaps even intercept correction.

Assuming that the econometric model coincides with the in-sample DGP is very strong, so practical application will not attain the precise offsets found here. Any empirical policy model will be invalid when it embodies the wrong causal attributions; its target-instrument links are not autonomous; or its parameters are not invariant to the policy change under analysis. These are distinct from the causes of forecast failure, though they could be a subset of the factors in any given situation. None of these problems need be revealed in-sample, but the failure of the policy to produce the anticipated results would do so, at a cost to society, offset by the benefits of improved knowledge of the economy. Nevertheless, the outcome seems unlike to be worse than using the either model alone. 


\section{References}

Banerjee, A., Hendry, D. F., and Mizon, G. E. (1996). The econometric analysis of economic policy. Oxford Bulletin of Economics and Statistics, 58, 573-600.

Barrell, R. (2001). Forecasting the world economy. in Hendry, and Ericsson (2001), pp. 149-169.

Bates, J. M., and Granger, C. W. J. (1969). The combination of forecasts. Operations Research Quarterly, 20, 451-468.

Bontemps, C., and Mizon, G. E. (2001). Congruence and encompassing. In Stigum, B. (ed.), Econometrics and the Philosophy of Economics. Cambridge, MA: MIT Press, forthcoming.

Boswijk, H. P. (1992). Cointegration, Identification and Exogeneity, Vol. 37 of Tinbergen Institute Research Series. Amsterdam: Thesis Publishers.

Britton, A. (ed.)(1989). Policy Making with Macroeconomic Models. Aldershot, UK: Gower.

Bryant, R., Hooper, P., and Mann, C. L. (eds.)(1993). Evaluating Policy Regimes: New Research in Empirical Macroeconomics. Washington, DC: Brookings Institution.

Budd, A. (1998). Economic policy, with and without forecasts. Bank of England Quarterly Bulletin, 38, 379-384.

Burns, T. (2001). The costs of forecast errors. in Hendry, and Ericsson (2001), pp. 170-184.

Clements, M. P., and Hendry, D. F. (1995). Forecasting in macroeconomics. In Cox, D., Hinkley, D., and Barndorff-Nielsen, O. (eds.), Time Series Models in Econometrics, Finance and Other Fields, pp. 99-138. London: Chapman and Hall.

Clements, M. P., and Hendry, D. F. (1996). Intercept corrections and structural change. Journal of Applied Econometrics, 11, 475-494.

Clements, M. P., and Hendry, D. F. (1998). Forecasting Economic Time Series. Cambridge: Cambridge University Press.

Clements, M. P., and Hendry, D. F. (1999a). Forecasting Non-stationary Economic Time Series. Cambridge, Mass.: MIT Press.

Clements, M. P., and Hendry, D. F. (1999b). On winning forecasting competitions in economics. Spanish Economic Review, 1, 123-160.

Clements, M. P., and Hendry, D. F. (2001). An historical perspective on forecast errors. National Institute Economic Review, 177, 100-112.

Doornik, J. A., Hendry, D. F., and Nielsen, B. (1998). Inference in cointegrated models: UK M1 revisited. Journal of Economic Surveys, 12, 533-572.

Granger, C. W. J., and Deutsch, M. (1992). Comments on the evaluation of policy models. Journal of Policy Modeling, 14, 497-516.

Harris, R. I. D. (1995). Using Cointegration Analysis in Econometric Modelling. London: Prentice Hall.

Hendry, D. F. (1981). Econometric evidence in the appraisal of UK monetary policy. In The Third Report of the Select Committee of the House of Commons on the Treasury and Civil Service, Vol. 3, pp. 1-21: HMSO.

Hendry, D. F. (1991). Economic forecasting. In Treasury and Civil Service Select Committee 
Memorandum: HMSO.

Hendry, D. F. (1995). Dynamic Econometrics. Oxford: Oxford University Press.

Hendry, D. F. (1997). The econometrics of macro-economic forecasting. Economic Journal, 107, 1330-1357. Reprinted in T.C. Mills (ed.), Economic Forecasting. Edward Elgar, 1999.

Hendry, D. F. (2000). On detectable and non-detectable structural change. Structural Change and Economic Dynamics, 11, 45-65.

Hendry, D. F., and Clements, M. P. (2000). Economic forecasting in the face of structural breaks. In Holly, S., and Weale, M. (eds.), Econometric Modelling: Techniques and Applications, pp. 3-37. Cambridge: Cambridge University Press.

Hendry, D. F., and Doornik, J. A. (1997). The implications for econometric modelling of forecast failure. Scottish Journal of Political Economy, 44, 437-461. Special Issue.

Hendry, D. F., and Ericsson, N. R. (1991). Modeling the demand for narrow money in the United Kingdom and the United States. European Economic Review, 35, 833-886.

Hendry, D. F., and Ericsson, N. R. (eds.)(2001). Understanding Economic Forecasts. Cambridge, Mass.: MIT Press.

Hendry, D. F., and Mizon, G. E. (1993). Evaluating dynamic econometric models by encompassing the VAR. In Phillips, P. C. B. (ed.), Models, Methods and Applications of Econometrics, pp. 272-300. Oxford: Basil Blackwell.

Hendry, D. F., and Mizon, G. E. (1998). Exogeneity, causality, and co-breaking in economic policy analysis of a small econometric model of money in the UK. Empirical Economics, 23, 267-294.

Hendry, D. F., and Mizon, G. E. (2000). On selecting policy analysis models by forecast accuracy. In Atkinson, A. B., Glennester, H., and Stern, N. H. (eds.), Putting Economics to Work. Volume in Honour of Michio Morishima, pp. 71-119. London: London School of Economics. STICERD Occasional Paper No. 22 ISBN 0753013991.

Johansen, S. (1992). Testing weak exogeneity and the order of cointegration in UK money demand. Journal of Policy Modeling, 14, 313-334.

Johansen, S., and Juselius, K. (2000). How to control a target variable in the VAR model. Mimeo, European University of Institute, Florence.

Lucas, R. E. (1976). Econometric policy evaluation: A critique. In Brunner, K., and Meltzer, A. (eds.), The Phillips Curve and Labor Markets, Vol. 1 of Carnegie-Rochester Conferences on Public Policy, pp. 19-46. Amsterdam: North-Holland Publishing Company.

Mizon, G. E. (1995). Progressive modelling of macroeconomic time series: the LSE methodology. In Hoover, K. D. (ed.), Macroeconometrics: Developments, Tensions and Prospects, pp. 107-169. Dordrecht: Kluwer Academic Press.

Paroulo, P. (1996). On the determination of integration indices in I(2) systems. Journal of Econometrics, 72, 313-356.

Rahbek, A., Kongsted, H. C., and Jørgensen, C. (1999). Trend-stationarity in the I(2) cointegration model. Journal of Econometrics, 90, 265-289.

Sims, C. A. (1982). Policy analysis with econometric models. Brookings Papers on Economic 
Activity, 1, 107-164.

Sims, C. A. (1986). Are forecasting models useful for policy analysis?. Federal Reserve Bank of Minneapolis Quarterly Review, Winter, 2-16.

Stock, J. H., and Watson, M. W. (1996). Evidence on structural instability in macroeconomic time series relations. Journal of Business and Economic Statistics, 14, 11-30.

Turner, D. S., Wallis, K. F., and Whitley, J. D. (1989). Using macroeconometric models to evaluate policy proposals. in Britton (1989). 